\title{
Tracking lexical consolidation with ERPs: Lexical and semantic-priming effects on N400 and LPC responses to newly-learned words
}

\author{
Iske Bakker $^{\mathrm{a}, \mathrm{b}, *}$, Atsuko Takashima ${ }^{\mathrm{a}, \mathrm{b}}$, Janet G. van Hell ${ }^{\mathrm{c}, \mathrm{a}}$, Gabriele Janzen ${ }^{\mathrm{a}, \mathrm{b}}$, \\ James M. McQueen ${ }^{\mathrm{a}, \mathrm{b}, \mathrm{d}}$ \\ a Radboud University Nijmegen, Behavioural Science Institute, P.O. Box 9104, 6500 HE Nijmegen, The Netherlands \\ ${ }^{\mathrm{b}}$ Radboud University Nijmegen, Donders Institute for Brain, Cognition and Behaviour, P.O. Box 9101, 6500 HB Nijmegen, The Netherlands \\ c Pennsylvania State University, Department of Psychology, University Park, PA 16802, USA \\ ${ }^{\mathrm{d}}$ Max Planck Institute for Psycholinguistics, Nijmegen, P.O. Box 310, 6500 AH Nijmegen, The Netherlands
}

\section{A R T I C L E I N F O}

\section{Article history:}

Received 1 December 2014

Received in revised form

29 July 2015

Accepted 12 October 2015

Available online 22 October 2015

Keywords:

Memory consolidation

Word learning

N400

LPC

EEG

Semantic priming

\begin{abstract}
A B S T R A C T
Novel words can be recalled immediately and after little exposure, but require a post-learning consolidation period to show word-like behaviour such as lexical competition. This pattern is thought to reflect a qualitative shift from episodic to lexical representations. However, several studies have reported immediate effects of meaningful novel words on semantic processing, suggesting that integration of novel word meanings may not require consolidation. The current study synthesises and extends these findings by showing a dissociation between lexical and semantic effects on the electrophysiological (N400, LPC) response to novel words. The difference in N400 amplitude between novel and existing words (a lexical effect) decreased significantly after a 24 -h consolidation period, providing novel support for the hypothesis that offline consolidation aids lexicalisation. In contrast, novel words preceded by semantically related primes elicited a more positive LPC response (a semantic-priming effect) both before and after consolidation, indicating that certain semantic effects can be observed even when words have not been fully lexicalised. We propose that novel meanings immediately start to contribute to semantic processing, but that the underlying neural processes may shift from strategic to more automatic with consolidation.
\end{abstract}

(c) 2015 Elsevier Ltd. All rights reserved.

\section{Introduction}

The human brain is able to extract and store lexical information from unfamiliar input extremely rapidly (Coutanche and Thompson-Schill, 2014; Saffran et al., 1997). Several studies have shown that a single exposure to a new word in a meaningful context can be enough to evoke neural signatures of semantic integration and processing (Borovsky et al., 2012, 2010). But evidence also suggests that the formation of a new lexical representation is far from complete after the initial learning event. Like non-linguistic memories, novel words are thought to undergo a post-learning consolidation process during which hippocampal connections decay and neocortical connections are strengthened (Frankland and Bontempi, 2005; McClelland et al., 1995). This shift towards neocortical storage underlies novel words' integration into the mental lexicon, allowing newly formed representations to interact

\footnotetext{
* Correspondence to: Donders Centre for Cognitive Neuroimaging, Kapittelweg 29, 6525 EN Nijmegen, The Netherlands.

E-mail address: i.bakker@donders.ru.nl (I. Bakker).
}

with existing information (Davis and Gaskell, 2009). Thus, truly word-like behaviour only emerges after a sufficient consolidation period.

Empirical support for this notion largely comes from paradigms designed to test the influence of novel words on the processing of existing words, under the assumption that interaction between words is a measure of novel words' integration into the lexical network. For example, consolidation has been shown to enable novel words to enter into competition for selection with their existing phonological and orthographic neighbours during word recognition (e.g. Bakker et al., 2014; Bowers et al., 2005; Dumay and Gaskell, 2007, 2012; Gaskell and Dumay, 2003). Most of these studies have focused on the integration of novel word forms into the mental lexicon, and used novel word stimuli without semantic content. The present EEG study examined whether offline consolidation also affects the lexicalisation of meaningful novel words, or whether semantic processing during encoding suffices to establish stable, integrated semantic representations.

As a marker of lexical access we used the N400 component, a negative-going event-related potential (ERP) peaking around 
$400 \mathrm{~ms}$ after stimulus onset (Kutas and Hillyard, 1980) and believed to reflect largely automatic processes of lexical-semantic retrieval (for discussion see Kutas and Federmeier, 2011). The N400 is especially suitable as a measure of lexical and semantic integration, as its amplitude is modulated by various linguistic properties. Firstly, pseudowords elicit more negative responses than real words (Bentin, 1987), thus providing a test of the lexical status of a newly-acquired word. The present study tested whether consolidation influences these lexical effects in the N400 response to novel words. Secondly, the N400 response is reduced (i.e., more positive) when the stimulus can be predicted based on the preceding context, for example a sentence or a semantically related prime word (Bentin et al., 1985; Kutas and Hillyard, 1980). The emergence of similar priming effects on N400 amplitude due to the combination of existing word primes and novel word targets would suggest semantic links between the two, that is, a degree of integration of novel words into the existing semantic network. Here, we examined whether semantic-priming effects on the N400 response to novel words change as a result of consolidation.

Both the lexical effect and the semantic-priming effect have been shown to emerge relatively early in second language learning. For example, McLaughlin et al. (2004) presented learners of French with a set of French words and pseudowords in a primed lexical decision task. After only $14 \mathrm{~h}$ of classroom instruction, subjects showed reduced N400 responses to real French words compared to pseudowords. This lexical N400 effect emerged despite chance-level behavioural performance, suggesting that ERP measures may reflect neural changes that are too subtle to be visible in behavioural measures such as reaction times or accuracy scores. In the second session, after 63 class hours, an additional semantic effect emerged: N400 responses to semantically related prime-target pairs (e.g. chien-CHAT, dog-CAT) were reduced relative to unrelated pairs (maison-SOIF, home-THIRST). Similar N400 priming effects in MEG data have been reported for novel words that were associated with pictures of existing objects through an implicit, multi-day statistical learning procedure (Dobel et al., 2010). These data suggest that both lexical and semantic N400 effects can be obtained in low-proficiency learners, but leave open the question of exactly how much exposure and postlearning consolidation is necessary for these effects to emerge.

This issue has been addressed in several studies using artificial word-learning paradigms with minimal training and no trainingtest delay (Borovsky et al., 2010, 2012; Mestres-Missé et al., 2007). In these studies, subjects inferred the meaning of a novel word from its sentence context. Even this rather implicit, restricted learning opportunity produced semantic N400 effects in priming tasks (Borovsky et al., 2012; Mestres-Missé et al., 2007) and during sentence processing (Borovsky et al., 2010), immediately after training. Similar priming effects were observed by Perfetti et al. (2005) and Balass et al. (2010) when meanings were acquired through explicit studying of definitions. These data suggest that rapid semantic integration had taken place without consolidation, allowing novel words to influence activation levels of semantically associated existing words immediately after acquisition.

It could be argued, however, that some or all of these rapid effects may have been driven by novel word representations that had not fully achieved lexical status (Tamminen and Gaskell, 2012). For instance, the target words in the related prime-target pairs often occurred in the novel word's definition (Perfetti et al., 2005) or were the direct translations of their novel word primes (Mestres-Missé et al., 2007). In such cases, priming may have arisen due to an episodic connection between the prime and target words. Given that relatively long SOA's and visible primes were used, strategic priming processes relying on episodic retrieval rather than lexical activation may have been the main factor contributing to the observed N400 effects (Kiefer and Spitzer, 2000).

For this reason, Tamminen and Gaskell (2012) examined whether novel words could prime semantically (but not episodically) related existing words in a masked-priming paradigm. When subjects were tested immediately after the training session, no behavioural priming effects emerged. In contrast, a small but significant reaction time benefit for related prime-target pairs was found when the training and test sessions were separated by a week. These findings suggest that automatic priming effects are affected by offline consolidation. However, the behavioural change between the immediate and delayed test sessions was minimal: although the priming effect did not reach significance in the immediate session, there was no significant interaction of primetarget relatedness and training-test interval. Furthermore, the possibility remains that the behavioural priming paradigm was not sensitive enough to register small semantic effects in the immediate test session, as suggested by the dissociation between behavioural and electrophysiological measures in McLaughlin et al. (2004).

The present study therefore investigated whether lexical and/ or semantic N400 effects change with offline consolidation. Subjects memorised two sets of novel words paired with definitions, one set on each of two consecutive days. Following the second session, N400 responses to words from both sets were measured. Thus, within the same recording session, novel words that had undergone a 24-h consolidation period could be directly compared to novel words that had been learned immediately before testing. The novel words served as targets that were preceded by either semantically related or unrelated existing word primes. This allowed us to measure both lexical (word versus pseudoword) and semantic (related versus unrelated prime) N400 effects in response to the novel words themselves rather than to their existing semantic associates. We predicted that novel words that had had an opportunity for offline consolidation would show a more wordlike (i.e., less negative) N400 response than words learned immediately before testing. In other words, consolidation was expected to decrease the lexical N400 effect for novel words. If this lexical effect goes hand-in-hand with the integration of semantic representations for novel words, then N400 amplitude for the consolidated words should also be modulated by the semantic relatedness of the prime words. Thus, the semantic-priming N400 effect would be expected to increase after consolidation.

Though it has not received the same amount of attention with respect to semantic context effects as the N400, a later positive component (LPC) peaking around $600 \mathrm{~ms}$ is usually observed following the N400 response to word stimuli. While the N400 is at least in part driven by automatic processes (Kutas and Federmeier, 2011), the LPC has been linked to more controlled, explicit semantic access (Hoshino and Thierry, 2012; Juottonen et al., 1996; Martin et al., 2009; Rohaut et al., 2015) and episodic memory retrieval (Rugg and Curran, 2007). Given that automatic access to fully lexicalised representations is presumably not necessary for strategic, overt priming effects to emerge, we may expect to see semantic-priming LPC effects for both consolidated and unconsolidated novel words.

\section{Methods}

\subsection{Participants}

Twenty-two right-handed (as assessed by an abridged version of the Edinburgh Handedness Inventory; Oldfield, 1971) native speakers of Dutch (6 males), aged 18-28 years (mean 21), participated in the experiment in return for course credit or monetary 
compensation. Participants had no history of neurological or language-related disorders, and reported having normal or correctedto-normal vision and normal hearing.

\subsection{Materials}

Four lists of 20 novel words of 5-7 letters (mean 5.9) were created by substituting one letter of an existing Dutch word, for example 'pamat' from 'patat' (chips). The substituted letter was in the first position in 9 words, between second and penultimate position in 59 words, and in last position in 12 words. Lists were matched on word length and on the lexical frequency of the existing neighbour (0-175 tokens per million words, mean 23.8) according to the CELEX database (Baayen et al., 1995).

Two lists of 20 definitions were created to provide the novel words' meanings, in part based on Tamminen and Gaskell (2012). Each definition consisted of an existing object category paired with two distinguishing features, for example 'A cat that has stripes and is bluish grey', thus describing a novel subcategory. For each participant, two lists of novel words and both lists of definitions served as the to-be-learned material, one in each of two learning sessions. The pairing of words and definitions was randomised for each participant.

Sixty existing Dutch words were divided across three lists of 20 words, matched on frequency (1-195 per million words, mean 33.6) and length (5-7 letters, mean 6). Each existing word was presented with a realistic definition (e.g. 'lemon: a yellow, sourtasting fruit'). Participants saw two of the three lists of existing words and definitions, one in each of the two learning sessions.

For the purpose of the semantic-priming task, two semantically related prime words per learned word were selected from a Dutch database of word associations (De Deyne and Storms, 2008) or, in case the prime word was unavailable in that database, from the Florida Free Association Norms (Nelson et al., 1998). The category name (e.g. 'cat') of the novel word definitions was used as the basis for selecting two associations (e.g. 'dog' and 'tail'). We selected the two cues that most often elicited the stimulus word as a response, and met our criteria (no longer than 7 letters, consisting of a single lemma, well-known, not occurring in the definition of their target, not a synonym of the target). The average forward association strength was .17 for novel prime-target pairs and .14 for existing pairs. Primes were existing Dutch words of 3-7 letters long (average 5) with CELEX frequencies between 1 and 882 per million (average 44). Two semantically unrelated primes were created for each target by pseudo-randomly reusing the related primes.

\subsection{Procedure}

The experiment consisted of two training sessions followed by a test phase (Fig. 1). In both sessions, participants learned a set of novel and existing words paired with a definition. Following the training phase in the second session, EEG responses were recorded while participants performed a priming task on pairs of untrained existing word primes and trained novel or existing word targets. This design allowed us to directly compare, within a single recording session, lexical and semantic effects on novel words learned before and after a consolidation period. The existing words that were trained identically to the novel words provided a baseline against which to assess the lexical N400 effect, keeping the level of recent exposure between novel and existing words constant.

For the purpose of testing word-form integration, a behavioural lexical decision task on the existing orthographic neighbours (e.g. 'patat' for 'pamat') was administered after the EEG session (for details see Bakker et al., 2015).

\subsubsection{Training}

The training phase in the first session started with a round of 5 -s visual presentations of each of the 20 novel and 20 existing words with their definitions. A series of four training tasks was then administered twice, in the following fixed order: (1) twoalternative-forced-choice (2AFC) word-definition matching, where definitions were the cues and words were the choices; 3 trials for each item; (2) 2AFC word-definition matching, where words were the cues and definitions were the choices; 3 trials for each item; (3) recall of words cued by definitions; and (4) recall of definitions cued by words. Thus, each word was presented 17 times in total. The order of items was randomised for each task. The incorrect word and definition options were all other items from the set. Participants gave their responses by pressing a key (in tasks 1 and 2) or typing on the keyboard (in tasks 3 and 4), after which the correct response was presented and remained on the screen for $3 \mathrm{~s}$. There was no response time limit. Participants returned after $24 \mathrm{~h}$ and received an identical training session with a different set of novel and existing words. Thus, at the time of testing on day 2, one set of words had been learned $24 \mathrm{~h}$ previously (the remote condition) and another set had been learned immediately before test (the recent condition).

\subsubsection{Memory tests}

Immediately after the training phase in the second session, an additional block of definition recall was presented without feedback, containing all words from both sessions. This block served both to assess memory and as a reactivation of the words in the remote condition in order to reduce any differences between conditions in perceptual processing of the words due to recency of exposure. At the end of the session, after the EEG recording, a block of 4AFC word-definition matching was administered. As in the training task, participants indicated which of four definitions from the trained set belonged to a target word. Since definition

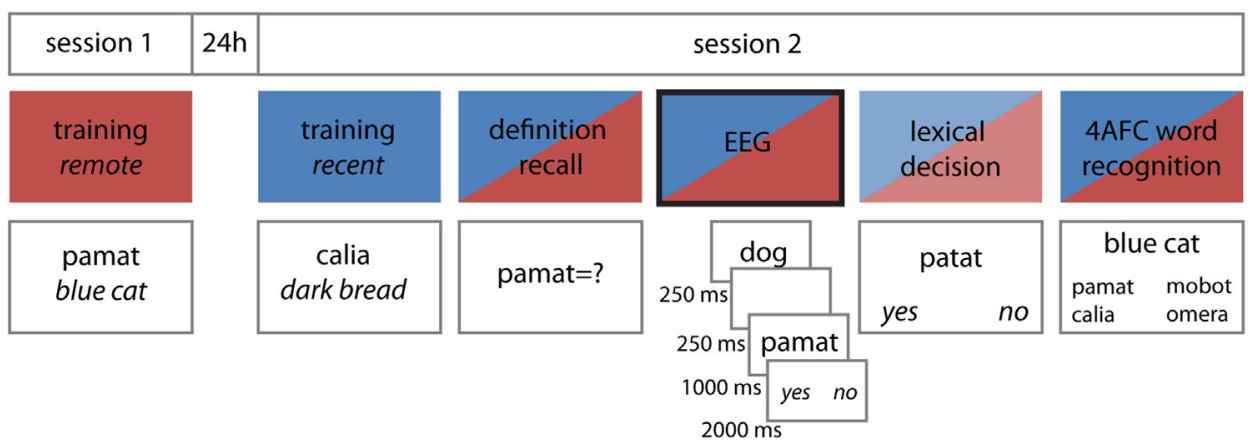

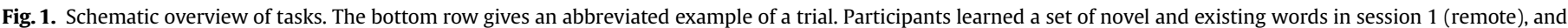
a second set of novel and existing words $24 \mathrm{~h}$ later in session 2 (recent). Tests on day 2 included both the recent and remote sets. 
recall is relatively difficult, especially for the remote condition, this easier task was included to confirm that learning of both sets had been successful and no excessive forgetting of the remote condition had occurred.

\subsubsection{EEG task}

The EEG session started after a short break and preparation of the recording, approximately one hour after completion of the second training session. It started with a task designed to measure oscillatory EEG responses, in which participants made natural/artefact decisions to all trained novel and existing words (for details see Bakker et al., 2015).

The priming task included all novel and existing words learned $24 \mathrm{~h}$ previously in session 1 (the remote condition) and those learned just before test in session 2 (the recent condition). Each novel and existing learned target word was presented four times; once with each of the two semantically related primes and once with each of the two unrelated primes (i.e., no prime-target pairs were repeated). Repetitions of the same targets were separated by at least 40 trials. A trial started with a $600 \mathrm{~ms}$ fixation screen. The prime was presented for $250 \mathrm{~ms}$, followed by a blank screen for $250 \mathrm{~ms}$, and the target for $1000 \mathrm{~ms}$. A response prompt then appeared and remained on the screen for $2000 \mathrm{~ms}$ or until a button was pressed. The participant was free to blink for a period of $1000 \mathrm{~ms}$ before the onset of the next trial.

For each prime-target pair, the participants' task was to decide whether the two words were semantically related or not by pressing one of two buttons. Semantic relatedness judgement has been shown to preserve priming effects better than lexical decision when stimuli are repeated (Renoult et al., 2012), which was necessary here given the limited set of primes. Furthermore, this task has the advantage of avoiding the response ambiguity that is problematic in lexical decision tasks with novel words (i.e., is the correct response to a new word 'yes', because the participant has learned it, or 'no', because it is clearly not a word in their native language?).

\subsection{EEG acquisition and preprocessing}

Continuous EEG was recorded from 58 electrodes spaced equidistantly on an Acticap systems cap, amplified with a BrainAmps DC amplifier ( $500 \mathrm{~Hz}$ sampling rate, $0.1-1000 \mathrm{~Hz}$ cut-off), referenced online to the left mastoid. EOG was recorded from two electrodes placed at the temples and two placed above and below the left eye. Impedances were kept below $20 \mathrm{k} \Omega$.

EEG preprocessing and analysis was done using the Fieldtrip toolbox (http://fieldtrip.fcdonders.nl/, Oostenveld et al., 2011). The signal was re-referenced offline to the averaged left and right mastoids and band-pass filtered at $.1-30 \mathrm{~Hz}$. Epochs of $100 \mathrm{~ms}$ before to $1000 \mathrm{~ms}$ after the onset of the target word were extracted. Trials containing muscle, eye-blink and other artefacts were removed manually (12\%) and noisy channels were interpolated using the averaged signal of neighbouring channels. Trials on which no or an incorrect behavioural response was given were rejected. Only participants with at least 20 trials in each condition (average 34 trials per condition) were considered for further analysis, which led to the rejection of two participants. ERPs were computed by averaging all remaining trials within each condition with a baseline of $100-0 \mathrm{~ms}$ before target onset. We analysed responses in a standard N400 time-window of 300-500 ms, and in a 500-700 ms window where LPC effects are typically observed.

\section{Results}

\subsection{Behavioural results}

In the definition recall test following the training phase in session 2, participants remembered significantly more features from the definitions of the recent (89.4\%) than of the remote words $(51.5 \% ; t(19)=10.987, p<.001)$. Recognition of words cued by their definitions in the 4AFC task at the end of session 2 was near ceiling for the remote as well as the recent condition $(97.9 \%$ remote, $98.2 \%$ recent). Note that in addition to passive recognition being easier than active recall, the recognition task may have also benefited from exposure to the novel words in the intervening EEG tasks. Nonetheless, this could have served only to reactivate existing memory traces, since no definitions were presented in the EEG tasks. Thus, these results suggest that although some forgetting had taken place, novel words had been learned successfully and memory representations of most words and their definitions were still retrievable after $24 \mathrm{~h}$.

Accuracy scores from the prime-target relatedness judgement task were analysed in a repeated-measures ANOVA with factors Lexicality (novel, existing), Day (recent, remote), and Relatedness (related, unrelated). Overall, performance was better for existing words ( $97 \% \pm 1$ correct for remote, $96 \% \pm 1$ for recent items) than novel words (remote: $82 \% \pm 2$, recent: $86 \% \pm 2$ ): $F(1,19)=58.42$, $p<.001$. Errors constituted more misses than false alarms ( $F$ $(1,19)=47.83, p<.001$ ), but only for novel words (as shown by the interaction Lexicality $\times$ Relatedness: $F(1,19)=55.99, p<.001)$. No main effect of Day was observed, but an interaction of Lexicality and Day indicated higher accuracy in the recent condition than in the remote condition for novel words only $(F(1,19)=7.15, p=.015)$. Thus, error rates suggested a small advantage for recently acquired novel words (Fig. 2A).

Reaction Time (RT) was measured from the response prompt, that is, from target offset. Errors and RTs more than 2 standard deviations from the mean were removed from analysis (14\%). RTs were submitted to a repeated-measures ANOVA with factors Lexicality (novel, existing), Day (recent, remote) and Relatedness (related, unrelated). A main effect of Lexicality $(F(1,19)=37.25$, $p<.001$ ) reflected faster responses to existing targets (mean and SD in ms: $342 \pm 14$ ) compared to novel targets (404 \pm 15$)$. Responses to related prime-target pairs $(359 \pm 13)$ were faster than those to unrelated pairs ( $388 \pm 15)$, as indicated by a main effect of Relatedness: $F(1,19)=16.96, p=.001$. A main effect of Day indicated faster responses to remote $(367 \pm 14)$ than to recent items $(381 \pm 13): F(1,19)=8.46, p=.009$. The latter effect was driven by a $27 \mathrm{~ms}$ difference for novel words, against $0 \mathrm{~ms}$ for existing words (an interaction of Day $\times$ Lexicality: $F(1,19)=8.26, p=.01$ ). These results indicate that although memory performance was better for recently acquired novel words, successfully remembered remote novel words were responded to more rapidly (Fig. 2B).

\subsection{ERP results}

Amplitudes were averaged across an early time-window typical for N400 effects (300-500 ms) and a later time-window corresponding to the LPC (500-700 ms). These values were submitted to an omnibus repeated-measures ANOVA with factors Lexicality (novel, existing), Day (recent, remote), Relatedness (related prime, unrelated prime), Laterality (left, midline, right), Anteriority (anterior, central, posterior), and Window (early, late) following conventional distributional analysis (Luck, 2005). GreenhouseGeisser adjusted statistics are reported where assumptions of sphericity were violated. We first discuss effects and follow-up analyses involving the factor Lexicality only, and then turn to the analysis of priming effects (i.e., involving the factor Relatedness). 
A

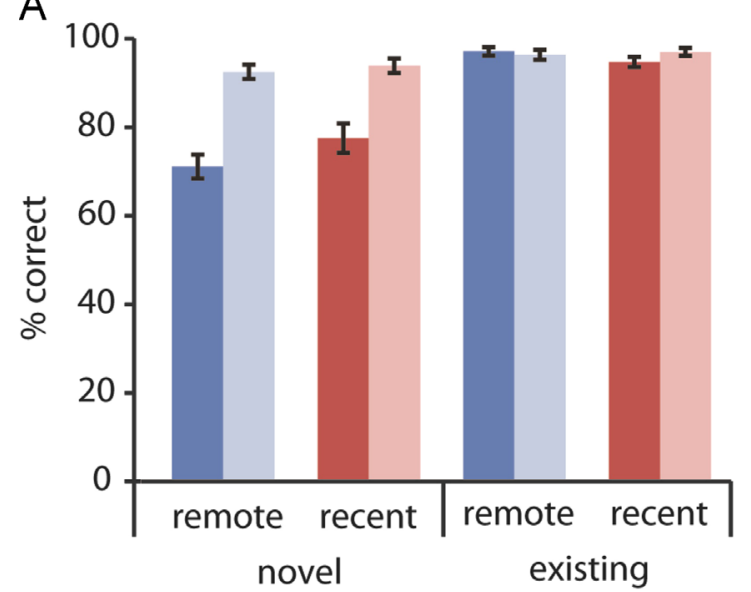

B

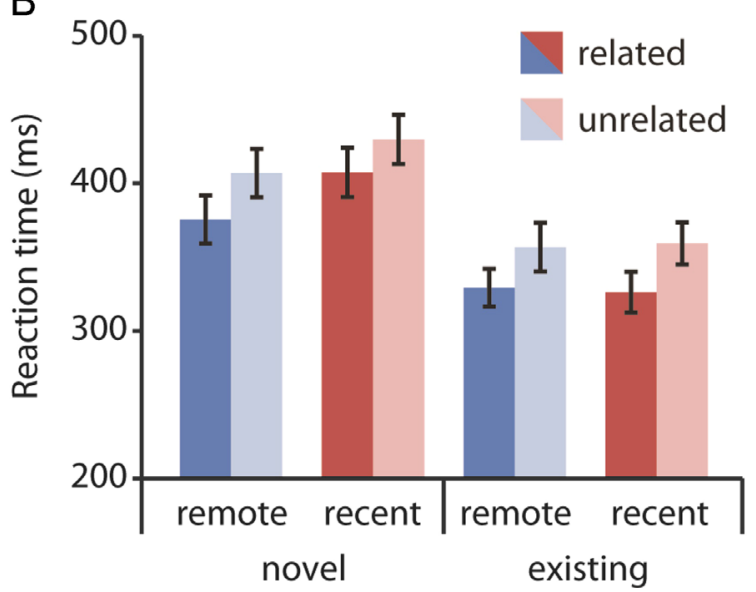

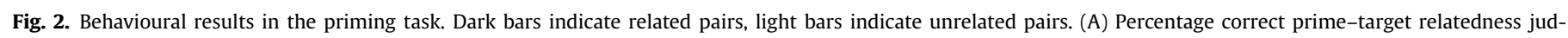

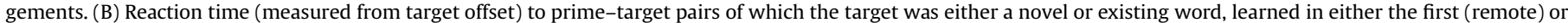
second (recent) session.

\subsubsection{Lexicality effects}

A main effect of Lexicality $(F(1,19)=101, p<.001)$ indicated a difference in amplitude between novel and existing words, with novel words eliciting more negative deflections than existing words (Fig. 3). This difference was largest over frontal and central medial electrodes (Lexicality $\times$ Hemisphere: $F(2,38)=58.608$, $p<.001$; Lexicality $\times$ Anteriority: $F(1.4,26.51)=18.297, p=.001$; Lexicality $\times$ Hemisphere $\times$ Anteriority: $F(4,76)=15.963, p<.001)$. Lexicality effects were more pronounced in the LPC time-window (Lexicality $\times$ Window: $F(1,19)=7.675, p=.012)$. Crucially, lexicality effects were larger for recent than remote words, as indicated by an interaction of Lexicality $\times$ Day: $F(1,19)=8.771, p=.008$.

To further specify the change in lexicality effects with consolidation, we performed ANOVAs with factors Day $\times$ Lexicality within the frontal and central midline ROIs that showed the largest overall effects, separately for each time window. Since the inclusion of the factor Relatedness may introduce a confound (because we also expect a larger amplitude reduction to the related prime-target pairs for the existing compared to the novel words), the following analyses were restricted to the unrelated trials.

3.2.1.1. N400 lexicality effects. In the $\mathrm{N} 400$ window, the frontal midline ROI did not exhibit main effects of Day, Lexicality, or an interaction. The central midline ROI showed no main effects but did reveal an interaction $(F(1,19)=8.088, p=.01)$, suggesting that the lexical N400 effect indeed decreased with consolidation. Paired $t$-tests revealed that remote novel words elicited responses similar to existing words $(t(19)=.811, p=.427)$ whereas the N400 to recent novel words was significantly more negative than to existing words $(t(19)=2.463, p=.024)$. A direct comparison of remote versus recent words furthermore confirmed the consolidation effect: this revealed a decrease in N400 for remote relative to recent novel words $(t(19)=2.86, p=.01)$, which, as expected, was not observed for existing words $(t(19)=.598, p=.557)$.

Each target was presented with two different unrelated and related primes. To investigate potential effects of target repetition on the N400, we repeated the ANOVA on the central midline ROI with Repetition as an additional factor. This revealed a main effect of Repetition $(F(1,19)=6.562, p=.019)$, reflecting more positive responses to the second trial, but Repetition did not interact with any other factor (Lexicality $\times$ Repetition: $F(1,19)=.13, p=.91$; Day$\times$ Repetition: $F(1,19)=.35, p=.561$; Lexicality $\times$ Day $\times$ Repetition: $F$ $(1,19)=1.085, p=.311)$. Furthermore, the significance levels of the paired $t$-tests comparing remote novel versus existing, recent novel versus existing, and remote versus recent novel words remained identical when taking only the first repetition of each trial into account.

3.2.1.2. LPC lexicality effects. In the LPC window, the frontal midline ROI exhibited main effects of both Lexicality $(F(1,19)=27.711$, $p<.001)$ and Day $(F(1,19)=5.005, p=.037)$ but no interaction
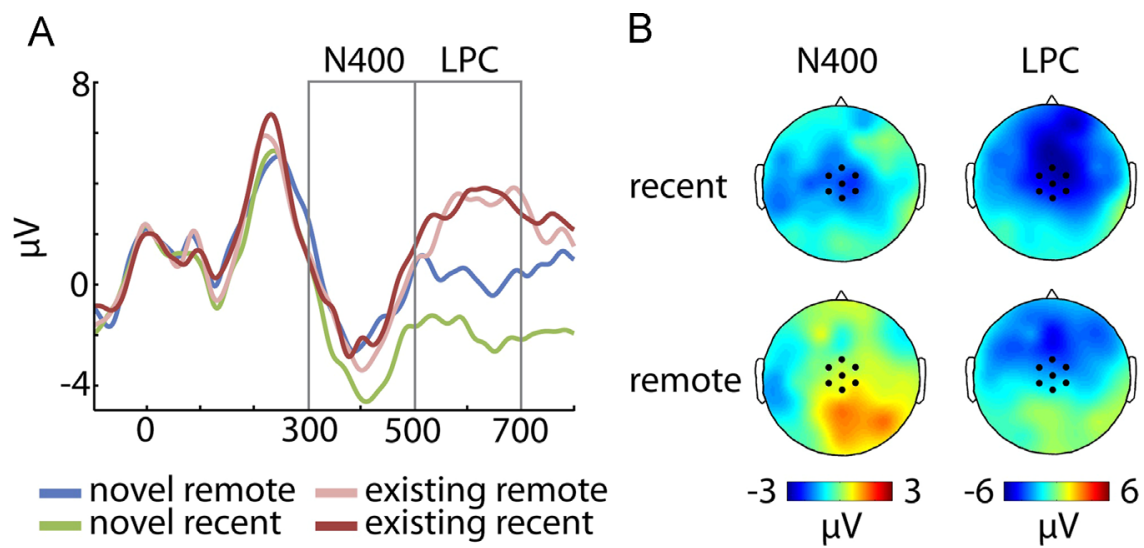

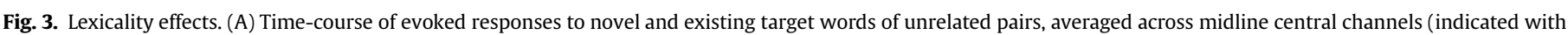

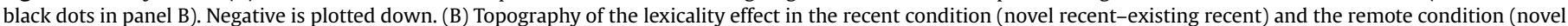
remote-existing remote), averaged across each time window. 
$(F(1,19)=1.182, p=.29)$. In the central midline ROI, we observed a main effect of Lexicality $(F(1,19)=14.056, p=.001)$, no main effect of Day $(F(1,19)=2.874, p=.106)$, and a marginally significant interaction $(F(1,19)=3.785, p=.067)$, reflecting a similar pattern as in the earlier time-window with lexicality effects being smaller for remote words. Paired $t$-tests showed that although both remote and recent words elicited more negative LPC responses than existing words (remote: $t(19)=2.35, p=.035$; recent: $t(19)=3.888$, $p=.001$ ), responses to remote novel words were more positive than those to recent words $(t(19)=2.148, p=.045)$. Existing words again did not exhibit this consolidation effect $(t(19)=.369$, $p=.716)$.

Adding Repetition as a factor to the central midline ROI again produced a main effect of Repetition $(F(1,19)=6.037, p=.024)$, but no interactions (Lexicality $\times$ Repetition: $F(1,19)=.678, p=.42$; Day$\times$ Repetition: $F(1,19)=.007, p=.936$; Lexicality $\times$ Day $\times$ Repetition: $F$ $(1,19)=.697, p=.414)$. The significance level of the paired $t$-tests did not change when repeating the analysis on the first-occurrence trials only, except for the comparison of novel and existing remote words which no longer reached significance $(t(19)=1.216, p=.239)$.

3.2.1.3. Summary. In sum, N400 responses to novel words over centro-parietal channels became more word-like after a 24-h consolidation period. The frontal LPC component exhibited lexicality effects that were not affected by consolidation, whereas the difference between novel and existing words was reduced in the central ROI. Remote novel words nonetheless still elicited more negative LPC responses than existing words, suggesting that the lexicalisation process had been set in motion but was not completed after $24 \mathrm{~h}$.

\subsubsection{Priming effects}

The omnibus ANOVA furthermore yielded a main effect of Relatedness $(F(1,19)=25.332, p<.001)$, confirming the expected reduction in N400 amplitude to targets following a related versus an unrelated prime (Fig. 4). This priming effect was larger for existing words than for novel words (Relatedness $\times$ Lexicality: $F(1,19)=6.81, p=.017$ ). A three-way interaction of Relatedness $\times$ Hemisphere $\times$ Anteriority reflected a typical N400 distribution that was maximal over central midline electrodes $(F(2.52,47.95)=7.561, p=.001)$. Priming effects for existing words were largest in the N400 window, whereas the priming effect for novel words reached its maximum in the LPC window (as shown by the interaction Relatedness $\times$ Lexicality $\times$ Window: $F(1,19)=14.302, p=.001)$.

An interaction of Relatedness $\times$ Day $\times$ Anteriority $(F(1.37$, $26.09)=11.047, p=.001$ ) reflected a pattern of larger priming effects for the recent condition in the frontal ROIs, and larger effects in the remote condition over central and posterior channels. While this anterior-posterior shift appeared to be stronger for novel words (see Fig. 4B), no interactions involving Lexicality, Relatedness and Anteriority reached significance.

3.2.2.1. N400 priming effects. To follow up these interactions, we conducted Day $\times$ Relatedness ANOVAs for novel and existing words, separately for each time-window and the three midline ROIs. For novel words in the N400 window, we observed main effects of Day in the frontal $(F(1,19)=4.834, p=.04)$, central $(F$ $(1,19)=14.053, p=.001)$, and posterior ROIs $(F(1,19)=18.951$, $p<.001)$. Main effects of Relatedness did not reach significance, although there was a trend in the central ROI $(F(1,19)=3.027$, $p=.098$ ). When Repetition was added as a factor, second-occurrence trials elicited more positive responses overall in the central and posterior ROIs (central: $F(1,19)=2.252, p=.034$; posterior: $F(1,19)=8.134, p=.01)$, but Repetition did not interact with any other factor (all $p>.067$ ).

Existing words exhibited main effects of Relatedness in the
A novel existing
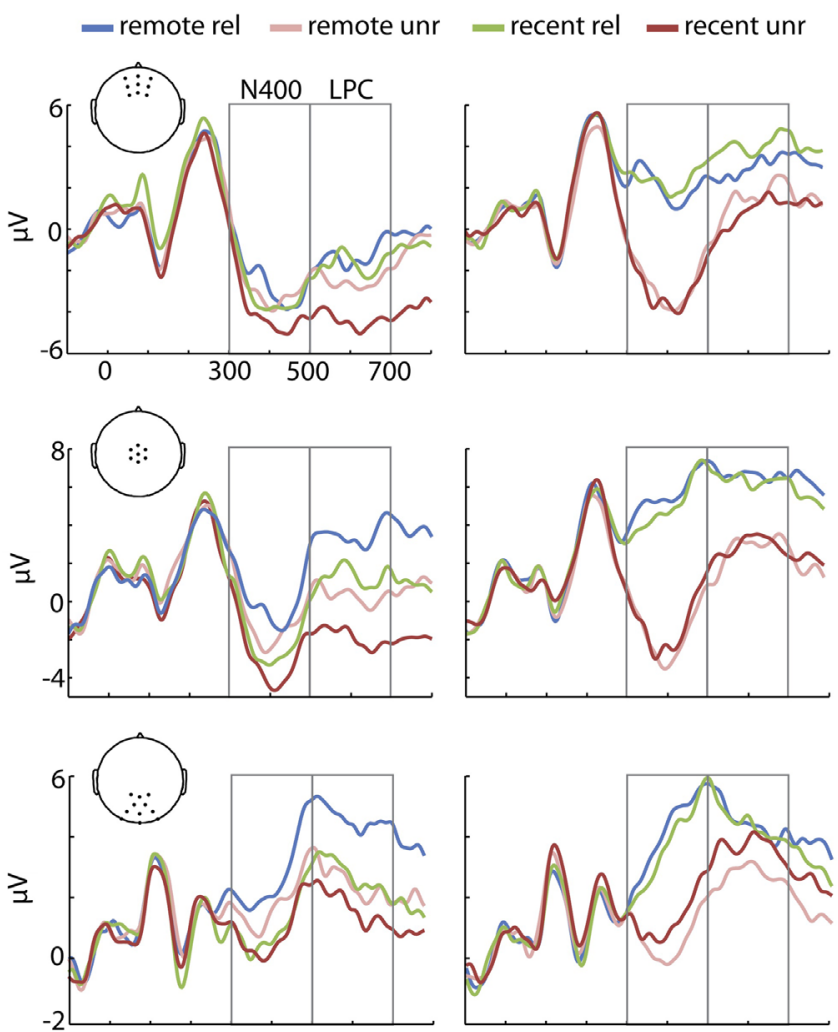

$\mathrm{B}$
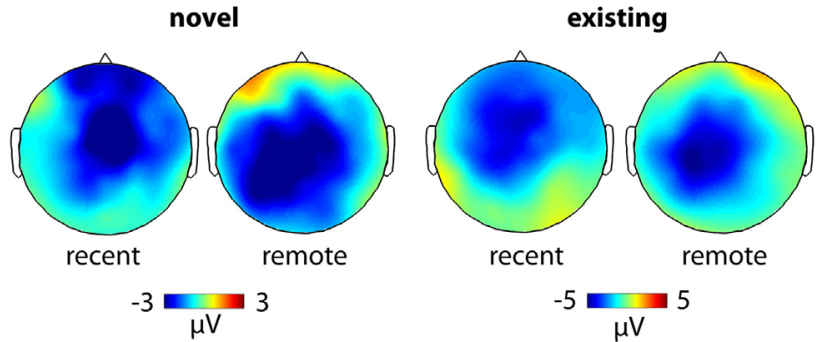

Fig. 4. Semantic priming effects. (A) Time-course of evoked responses to target words preceded by either a semantically related or unrelated existing prime, for novel words (left) and existing words (right). Responses are averaged across the frontal ROI (first row), central ROI (second row), and posterior ROI (third row) Negative is plotted down. (B) Topography of the priming effect (unrelated-related) in the LPC window.

N400 window in all three midline ROIs (frontal: $F(1,19)=39.225$, $p<.001$; central: $F(1,19)=62.165, p<.001$; posterior: $F(1,19)=$ $47.385, p<.001)$. There were no main effects of Day, however in the posterior ROI we did observe an interaction $(F(1,19)=7.993$, $p=.011$ ) reflecting larger priming effects in the remote condition. Paired $t$-tests following up this interaction showed that priming effects were nonetheless significant in both conditions (remote: $t$ $(19)=7.342, p<.001$; recent: $t(19)=5.152, p<.001)$. No main effects of Repetition or interaction with other factors were significant (all $p>.067$ ).

3.2.2.2. LPC priming effects. For novel words in the LPC window, the frontal ROI showed a trend towards a main effect of Day $(F(1,19)=3.165, p=.091)$ and a marginal effect of Relatedness $(F(1,19)=4.087, p=.058)$. Both main effects were significant in the central ROI (Day: $F(1,19)=8.907, p=.008$; Relatedness: $F(1,19)=$ $10.562, p=.004$ ), and in the posterior ROI (Day: $F(1,19)=15.485$, $p=.001$; Relatedness: $F(1,19)=7.116, p=.015)$, but the interactions 
did not reach significance. There were no main effects or interactions with Repetition (all $p>.08$ ).

In line with the interaction of Relatedness $\times$ Day $\times$ Anteriority in the omnibus ANOVA, paired $t$-tests showed priming effects for the recent condition $(t(19)=2.253, p=.036)$ but not the remote condition $(t(19)=1.045, p=.309)$ in the frontal ROI. Both conditions were significant in the central ROI (remote: $t(19)=2.587$, $p=.018$; recent: $t(19)=2.69, p=.014)$. In the posterior ROI the remote condition reached significance $(t(19)=2.748, p=.013)$, but the recent condition did not $(t(19)=1.489, p=.153)$. This suggests that priming effects were supported more by posterior regions in the remote condition and by frontal regions in the recent condition.

For existing words in the LPC window, main effects of Relatedness were seen in the frontal $(F(1,19)=4.695, p=.043)$, central $(F$ $(1,19)=12.613, p=.002)$ and posterior $(F(1,19)=5.131, p=.035)$ ROIs. A hint of an interaction reflecting larger priming effects in the recent condition was visible in the frontal ROI $(F(1,19)=3.179$, $p=.091)$. Paired $t$-tests showed significant priming in the recent condition $(t(19)=2.695, p=.014)$ but not the remote condition $(t$ $(19)=1.332, p=.199)$. The posterior ROI in contrast exhibited a trend towards increased priming in the remote condition ( $F$ $(1,19)=3.48, p=.078)$. Here, $t$-tests revealed significant priming in the remote condition $(t(19)=2.782, p=.012)$ but not the recent condition $(t(19)=1.294, p=.211)$. There were no main effects or interactions with Repetition.

3.2.2.3. Summary. Existing words elicited both reduced $\mathrm{N} 400$ and LPC responses when preceded by semantically related existing word primes, whilst the priming effect for novel words was only reliable in the later LPC window. The interval between acquisition and test of novel words did not affect the magnitude of this effect. We observed a shift towards more posterior effects in the remote condition, but this pattern was also visible for existing words.

\section{Discussion}

The present study examined whether offline consolidation affects the lexical and semantic integration of meaningful novel words. We hypothesised that if consolidation entails a fundamental change in the nature of novel word representations, neural responses to those items should exhibit more word-like patterns after a consolidation period of $24 \mathrm{~h}$. Novel words initially exhibited a lexical N400/LPC effect: recently learned novel words elicited overall larger negative deflections than existing words. Offline consolidation diminished this effect in the N400 window, with remotely learned novel words no longer eliciting significantly different responses from existing words over centro-parietal channels. Recently learned novel words thus behaved like pseudowords, whereas consolidated novel words patterned more closely with existing words. Although the centro-parietal LPC response to novel words likewise became more positive after consolidation, it remained reduced relative to existing words, indicating that lexicalisation was not yet complete after $24 \mathrm{~h}$. This pattern of results suggests that novel word representations and the processes controlling their retrieval indeed became more lexical in nature, consistent with the notion that offline consolidation promotes the gradual integration of novel words into the mental lexicon.

The more negative N400 and LPC responses to recently learned novel words, behaving like pseudowords, can be related to findings by Holcomb et al. (2002). They observed that pseudowords elicited more negative N400/LPC responses than words, and also that both words and pseudowords with many orthographic neighbours elicited more negative responses than those from sparse neighbourhoods. This can be understood by assuming that the ERP reflects the activation of multiple word candidates. When the input is a real word, it rapidly wins the lexical competition process and activation of the competitors is inhibited, leading to the termination of the N400. In the case of a pseudoword, in contrast, competitors remain active, allowing the ERP to continue for longer. This model may account for the current finding that consolidation leads to more positive N400/LPC responses, on the assumption that consolidated words are more likely to enter into lexical competition with their existing neighbours (e.g. Bakker et al., 2014; Bowers et al., 2005; Dumay and Gaskell, 2007, 2012; Gaskell and Dumay, 2003). A novel word from the recent condition will activate its existing neighbours, which then continue to contribute to the ERP response. A consolidated novel word, like a real word, will inhibit its neighbours and win the competition, thus terminating the negative deflection. Thus, consolidation results in a more word-like retrieval process during which novel words interact with their existing neighbours.

According to the standard model of consolidation, the various components of an event, such as the visual presentation of a word, are rapidly processed in the neocortex and bound into a coherent episode by the hippocampal system (Alvarez and Squire, 1994). During offline consolidation, especially during sleep (Mölle and Born, 2011; Stickgold and Walker, 2005), synchronised reactivation of the neocortical memory components gradually strengthens cortico-cortical connections between these new components and existing memory traces. Hippocampal-neocortical connections in contrast decay over time, until eventually the memory is stored entirely neocortically and its retrieval no longer involves the hippocampus (Frankland and Bontempi, 2005). In humans, this representational shift over time can be clearly observed in terms of relative activation levels during retrieval in the hippocampus and neocortical memory-related areas such as the medial prefrontal cortex (Smith and Squire, 2009; Takashima et al., 2006).

Recent evidence suggests that it is this consolidation mechanism, in particular the emergence of strong cortico-cortical connections between new and old representations, that underlies the lexicalisation of novel words. For example, in an fMRI study (Takashima et al., 2014) we investigated functional connectivity while subjects listened to words they had learned $24 \mathrm{~h}$ previously. The magnitude of lexical competition effects between novel words and their phonological neighbours correlated with the strength of connectivity between the auditory cortex and the left posterior middle temporal gyrus (pMTG), an area known to be involved in lexical retrieval. Rather than storing information locally, the pMTG is thought to represent mappings between widely distributed memory traces representing the orthographic, phonological, semantic and syntactic properties of words (Gow, 2012; Hickock and Poeppel, 2007; Lau et al., 2008). The neural basis of lexicalisation may thus be a language-specific instance of the general mechanism by which neocortical areas take over the binding function of the hippocampus, with the PMTG in particular serving as a lexical hub that maps form onto meaning.

Source localisation of the N400 and its magnetic counterpart has implicated the left pMTG as a main generator of the ERP/ERF, suggesting that the N400 reflects the activation of such lexical representations (for review, see Lau et al., 2008). In support of this view, fMRI studies have consistently revealed priming effects in the pMTG (Lau et al., 2008). Though the present sensor-level data cannot speak directly to the source of the observed lexicality effects, the finding that responses to novel words in the N400 window became increasingly positive over time fits well with the hypothesis that offline consolidation enables the formation of neocortical lexical representations in the pMTG. When a recently learned novel word is perceived, the hippocampus serves to activate associated memory traces representing its meaning, 
phonology, and possibly episodic details such as the context in which it was learned. In contrast, a word learned before a consolidation period may rely relatively more on the pMTG to activate its distributed semantic content and other information. Future studies employing high-density EEG or MEG would be instrumental in confirming this putative role of the pMTG in the generation of N400 responses to novel words.

The LPC, in contrast to the N400, has been claimed to reflect controlled, non-automatic processes of semantic retrieval (Hoshino and Thierry, 2012; Juottonen et al., 1996; Martin et al., 2009; Rohaut et al., 2015). Hoshino and Thierry (2012) and Martin et al. (2009) found that word meanings in the non-attended language of bilinguals modulated N400 but not LPC responses, whilst the attended language elicited priming effects in both windows. They conclude that the LPC reflects conscious semantic access and evaluation, which does not occur in the non-attended language. This interpretation is supported by the recent finding of Rohaut et al. (2015) that whereas N400 responses were present in both minimally conscious and vegetative-state patients, LPC modulations were only observable in the minimally conscious group. The persistent lexicality effect on the LPC may indicate a failure to explicitly retrieve each novel word meaning on some trials, given that for each subject a portion of the word-definition pairings only had weak representation or may have been completely forgotten, thereby decreasing the subject- and trial-averaged ERP. Additionally, the more positive LPC for existing words may reflect a consistent difference between novel and existing words on each trial, in each subject. For example, one inherent difference is that the set of semantic features associated with existing words was always richer compared to novel words (regardless of their memory strength), because novel word meanings (unlike those for existing words) consisted only of the information contained in their short definitions.

A semantic-priming N400/LPC effect, a reduction in amplitude of the response to words that are preceded by a semantically related prime, was observed both before and after the consolidation period. Both windows showed qualitatively similar patterns, but in the window-specific analyses only the LPC effects were reliable. Given that the recently learned novel words elicited a clear lexicality effect, this indicates that the semantic effect does not rely on fully lexicalised representations. A possible explanation is that the overt prime presentation and medium-length SOA allowed episodic memory traces of novel words to be activated by a strategic, controlled retrieval process. For example, the subject may be able to make a deliberate prediction of what the target word could be or at least of what it might mean, based on the meaning of the prime. This account would explain why priming effects emerged mainly in the LPC window, which has been implicated in explicit semantic retrieval (Hoshino and Thierry, 2012; Juottonen et al., 1996; Martin et al., 2009; Rohaut et al., 2015). A controlled priming process could take place regardless of which memory system episodic or lexical - currently binds the relevant memory traces. It is possible that the $\mathrm{N} 400$ priming effects that have been observed immediately after little training (Borovsky et al., 2010, 2012; Mestres-Missé et al., 2007; Perfetti et al., 2005) could at least in part be explained by explicit semantic retrieval of non-lexicalised representations (though note that these effects emerged earlier than our effects). As well as the possible contribution of strategic factors to the N400 itself (Kiefer and Spitzer, 2000), these effects may reflect the influence of a partially overlapping LPC on N400 amplitude (Hill et al., 2002). Future studies aimed at minimising strategic factors are clearly needed to assess the relative contributions of automatic and controlled processes to these effects.

If the contribution of the pMTG in the binding of a recently learned word is still relatively minimal, we may however expect a difference in the relative contribution of areas that generate the
N400/LPC even when priming effects on the ERP amplitude are visible for both consolidated and unconsolidated words. For instance, the inferior frontal gyrus (IFG) has been reported to show priming effects in fMRI at long, but not short SOA's (Lau et al., 2008). This suggests that the IFG is involved in strategic priming processes, which are thought to dominate at longer SOA's (Neely, 1991). Interestingly, the semantic LPC effect in our recent condition appeared to have a more frontal distribution than in the remote condition: priming effects in the frontal ROI were significant for the recent condition only, and in the posterior ROI for the remote condition only. Though these scalp level data do not permit direct conclusions about the generators of the signals, this pattern is consistent with two previous findings suggesting that distributional differences reflect distinct underlying mechanisms.

First, the anterior-posterior shift is in line with a previously reported similar distributional difference in N400 priming effects for novel and existing words. Using current source density mapping, Mestres-Missé et al. (2007) showed that a temporal source most likely contributed to N400 priming effects elicited by existing word primes, whereas the effects from novel words were estimated to originate in frontal regions. As argued by these authors, priming effects between existing words can rely on strong, stable links that enable automatic spreading activation, while novel word processing involves more cognitive control. The same distinction applies to our recent and remote novel words: due to increased neocortical integration after consolidation, priming effects in the remote condition are likely to be influenced to a larger extent by automatic processes than those in the recent condition.

Second, topographical differences in the LPC window have been linked to episodic memory retrieval (Johnson et al., 2008; Peters and Daum, 2009; Yick and Wilding, 2008). For instance, Peters and Daum (2009) studied LPC responses during retrieval of (known) words that had been paired with either a sound, a picture of a scene, or a picture of a face. Posterior LPC responses were more positive for items that were correctly remembered, regardless of their associated information. However, frontal LPC responses additionally distinguished between the three types of source memory, both in amplitude and lateralisation. The authors propose that the frontal LPC indexes episodic memory retrieval, whereas the parietal response reflects more general retrieval. Our observation of frontal priming effects for recent words thus fits well with the idea that these representations are more episodic in nature than those in the remote condition, and may drive strategic but not fully automatic priming. The fact that existing words also exhibited this frontal shift furthermore suggests that the relative contribution of automatic and strategic processes is dynamic and can be influenced by recent exposure, even when stable lexical links are available. An important aim for future work therefore is to determine the point in the lexicalisation trajectory at which automatic activation surpasses strategic factors in contributing to the priming effect, in order to further elucidate semantic aspects of novel word consolidation.

In conclusion, the present study showed that N400/LPC responses to novel words become more word-like after a 24-h consolidation period. This is consistent with the idea that underlying the lexicalisation of novel words is a shift from the hippocampus to neocortical areas (presumably the left pMTG in particular; Lau et al., 2008) in coding the mapping between word form and meaning. In addition, controlled retrieval processes, possibly supported by the IFG, appear to enable semantic priming even when words are not fully lexicalised. The current data thus suggest that whilst a qualitative change in lexical representation takes place during consolidation, the contribution of novel word meanings to semantic processing is a gradual process with multiple neural substrates. 


\section{Acknowledgements}

This research is funded by The Netherlands Organization for Scientific Research (NWO) Brain and Cognition Grant no. 433-09239. The authors would like to thank Dorothee Chwilla for advice on data analysis.

\section{References}

Alvarez, P., Squire, L.R., 1994. Memory consolidation and the medial temporal lobe: a simple network model. Proc. Natl. Acad. Sci. USA 91 (15) 7041-7015.

Baayen, R.H., Piepenbrock, R., Gulikers, L., 1995. The CELEX lexical database [webcelex]. University of Pennsylvania Linguistic Data Consortium, Philadelphia, PA

Bakker, I., Takashima, A., van Hell, J.G., Janzen, G., McQueen, J.M., 2014. Competition from unseen or unheard novel words: lexical consolidation across modalities. J. Mem. Lang. 73, 116-130.

Bakker, I., Takashima, A., van Hell, J.G., Janzen, G., McQueen, J.M., 2015. Changes in theta and beta oscillations as signatures of novel word consolidation. J. Cogn. Neurosci. 27 (7), 1286-1297.

Balass, M., Nelson, J.R., Perfetti, C.A., 2010. Word learning: an ERP investigation of word experience effects on recognition and word processing. Contemp. Educ. Psychol. 35 (2), 126-140.

Bentin, S., 1987. Event-related potentials, semantic processes, and expectancy factors in word recognition. Brain Lang. 31 (2), 308-327.

Bentin, S., McCarthy, G., Woods, C.C., 1985. Event-related potentials, lexical decision and semantic priming. Electroencephalogr. Clin. Neurophysiol. 60, 343-355.

Borovsky, A., Kutas, M., Elman, J.L., 2010. Learning to use words: event related potentials index single-shot contextual word learning. Cognition 116 (2), 289-296.

Borovsky, A., Elman, J.L., Kutas, M., 2012. Once is enough: N400 indexes semantic integration of novel word meanings from a single exposure in context. Lang. Learn. Dev. 8 (3), 278-302.

Bowers, J.S., Davis, C.J., Hanley, D.A., 2005. Interfering neighbours: the impact of novel word learning on the identification of visually similar words. Cognition 97 (3), B45-B54.

Coutanche, M.N., Thompson-Schill, S.L., 2014. Fast mapping rapidly integrates information into existing memory networks. Journal of Experimental Psychology: General 143 (6), 2296-2303.

Davis, M.H. Gaskell, M.G. 2009. A complementary systems account of word learning: neural and behavioural evidence. Philos. Trans. R. Soc. Lond. Ser. B Biol. Sci. 364 (1536), 3773-3800.

De Deyne, S., Storms, G., 2008. Word associations: norms for 1,424 Dutch words in a continuous task. Behav. Res. Methods 40 (1), 198-205.

Dobel, C., Junghöfer, M., Breitenstein, C., Klauke, B., Knecht, S., Pantev, C., Zwitserlood, P., 2010. New names for known things: on the association of novel word forms with existing semantic information. J. Cogn. Neurosci. 22 (6), 1251-1261.

Dumay, N., Gaskell, M.G., 2007. Sleep-associated changes in the mental representation of spoken words. Psychol. Sci. 18, 35-39.

Dumay, N., Gareth Gaskell, M., 2012. Overnight lexical consolidation revealed by speech segmentation. Cognition 123 (1), 119-132.

Frankland, P.W., Bontempi, B., 2005. The organization of recent and remote memories. Nat. Rev. Neurosci. 6 (2), 119-130.

Gaskell, M.G., Dumay, N., 2003. Lexical competition and the acquisition of novel words. Cognition 89 (2), 105-132.

Gow, D.W., 2012. The cortical organization of lexical knowledge: a dual lexicon model of spoken language processing. Brain Lang. 121 (3), 273-288.

Holcomb, P.J., Grainger, J., O’Rourke, T., 2002. An electrophysiological study of the effects of orthographic neighborhood size on printed word perception. Journa of Cognitive Neuroscience 14 (6), 938-950.

Hickok, G., Poeppel, D., 2007. The cortical organization of speech processing. Nat. Rev. Neurosci. 8, 393-402.

Hill, H., Strube, M., Roesch-Ely, D., Weisbrod, M., 2002. Automatic vs. controlled processes in semantic priming - Differentiation by event-related potentials. International Journal of Psychophysiology 44, 197-218.

Hoshino, N., Thierry, G., 2012. Do Spanish-English bilinguals have their fingers in two pies-or is it their toes? An electrophysiological investigation of semantic access in bilinguals. Front. Psychol. 3, 1-6.

Johnson, J.D., Minton, B.R., Rugg, M.D., 2008. Content dependence of the electrophysiological correlates of recollection. Neurolmage 39 (1), 406-416.
Juottonen, K., Revonsuo, A., Lang, H., 1996. Dissimilar age influences on two ERP waveforms (LPC and N400) reflecting semantic context effect. Cogn. Brain Res. 4, 99-107.

Kiefer, M., Spitzer, M., 2000. Time course of conscious and unconscious semantic brain activation. NeuroReport 11 (11), 2401-2407.

Kutas, M., Hillyard, S.A., 1980. Reading senseless sentences: brain potentials reflect semantic incongruity. Science 207, 203-205.

Kutas, M., Federmeier, K.D., 2011. Thirty years and counting: finding meaning in the N400 component of the event-related brain potential (ERP). Annu. Rev. Psychol. 62, 621-647.

Luck, S.J., 2005. Introduction to the event-related potential technique. MIT Press., Cambridge, MA

Lau, E.F., Phillips, C., Poeppel, D., 2008. A cortical network for semantics: (de)constructing the N400. Nat. Rev. Neurosci. 9 (12), 920-933.

Mölle, M., Born, J., 2011. Slow oscillations orchestrating fast oscillations and memory consolidation. Prog. Brain Res. 193, 93-110.

Martin, C.D., Dering, B., Thomas, E.M., Thierry, G., 2009. Brain potentials reveal semantic priming in both the "active" and the "non-attended" language of early bilinguals. NeuroImage 47 (1), 326-333.

McClelland, J.L., McNaughton, B.L., O'Reilly, R.C., 1995. Why there are complementary learning systems in the hippocampus and neocortex: insights from the successes and failures of connectionist models of learning and memory. Psychol. Rev. 102 (3), 419-457.

McLaughlin, J., Osterhout, L., Kim, A., 2004. Neural correlates of second-language word learning: minimal instruction produces rapid change. Nat. Neurosci. 7 (7), 703-704.

Mestres-Missé, A., Rodriguez-Fornells, A., Münte, T.F., 2007. Watching the brain during meaning acquisition. Cereb. Cortex 17 (8), 1858-1866.

Neely, J.H., 1991. Semantic priming effects in visual word recognition: A selective review of current findings and theories. In: Besner, D., Humpreys, G.W. (Eds.) Basic Processes in Reading: Visual Word Recognition. Lawrence Erlbaum Associates, Hillsdale, NJ, pp. 264-336.

Nelson, D.L., McEvoy, C.L., Schreiber, T.A., 1998. The University of South Florida Word Association, Rhyme, and Word Fragment Norms. Retrieved from: http:// www.usf.edu/FreeAssociation/.

Oldfield, R.C., 1971. The assessment and analysis of handedness: the Edinburgh inventory. Neuropsychologia 9 (1), 97-113.

Oostenveld, R., Fries, P., Maris, E., Schoffelen, J.-M., 2011. FieldTrip: open source software for advanced analysis of MEG, EEG, and invasive electrophysiological data. Comput. Intell. Neurosci. 2011, article ID 156869.

Perfetti, C., Wlotko, E.W., Hart, L.A., 2005. Word learning and individual differences in word learning reflected in event-related potentials. J. Exp. Psychol. - Learn. Mem. Cogn. 31 (6), 1281-1292.

Peters, J., Daum, I., 2009. Frontal but not parietal positivity during source recollection is sensitive to episodic content. Neurosci. Lett. 454 (3), 182-186.

Renoult, L., Wang, X., Mortimer, J., Debruille, J.B., 2012. Explicit semantic tasks are necessary to study semantic priming effects with high rates of repetition. Clin. Neurophysiol. 123 (4), 741-754.

Rohaut, B., Faugeras, F., Chausson, N., King, J.-R., Karoui, I. El, Cohen, L., Naccache, L., 2015. Probing ERP correlates of verbal semantic processing in patients with impaired consciousness. Neuropsychologia 66, 279-292.

Rugg, M.D., Curran, T., 2007. Event-related potentials and recognition memory. Trends Cogn. Sci. 11 (6), 251-257.

Saffran, J.R., Newport, E.L., Aslin, R.N., Tunick, R.A., Barrueco, S., 1997. Incidental language learning: listening (and learning) out of the corner of your ear. Psychol. Sci. 8 (2), 101-105.

Smith, C.N., Squire, L.R., 2009. Medial temporal lobe activity during retrieval of semantic memory is related to the age of the memory. J. Neurosci. 29 (4), 930-938.

Stickgold, R., Walker, M.P., 2005. Memory consolidation and reconsolidation: what is the role of sleep? Trends Neurosci. 28 (8), 408-415.

Takashima, A., Bakker, I., van Hell, J.G., Janzen, G., McQueen, J.M., 2014. Richness of information about novel words influences how episodic and semantic memory networks interact during lexicalization. NeuroImage 84, 265-278.

Takashima, A., Petersson, K.M., Rutters, F., Tendolkar, I., Jensen, O., Zwarts, M.J., McNaughton, B.L., Fernández, G., 2006. Declarative memory consolidation in humans: a prospective functional magnetic resonance imaging study. Proc. Natl. Acad. Sci. USA 103 (3), 756-761.

Tamminen, J., Gaskell, M.G., 2012. Novel word integration in the mental lexicon: evidence from unmasked and masked semantic priming. Q. J. Exp. Psychol. 60 (5), 1001-1025.

Yick, Y.Y., Wilding, E.L., 2008. Material-specific neural correlates of memory retrieval. Neuroreport 19 (15), 1463-1467. 